\author{
Editorial: \\ Introducing the Australasian fournal of Logic \\ Greg Restall \\ Philosophy Department, \\ The University of Melbourne \\ restallounimelb.edu.au \\ Published July I, 2003 \\ http://www.philosophy .unimelb.edu.au/ajl/2003 \\ (c) 2003 Greg Restall
}

Established in 2003, the Australasian Fournal of Logic is a fully refereed, freely available electronic journal covering all areas of research in logic. We aim to be a repository of timely, original and significant research in pure logic, and logic as it is applied in mathematics, computer science, linguistics and philosophy.

\title{
I WhAT IS IT?
}

SCOPE The AJL aims to cover all areas of pure and applied logic. This means that we solicit manuscripts in the central areas of logic (including, but not restricted to, the proof theory and model theory of particular logical systems), but also their application to related fields, such as mathematics (e.g. set theory, the model theory of particular mathematical theories, the lambda calculus, etc.), computer science (e.g. the formal analysis of computability and computation, formal semantics of programs and functions, etc.), linguistics (e.g. theories of types applied to grammars, formal models for syntactic and semantic structures, etc.) and philosophy (e.g. formal treatments of theories of meaning, truth and reference; the paradoxes of self-reference and vagueness; discussions of the significance and interpretation of formal theories; etc.). We aim to publish original, timely and significant research in these areas, with an eye to furthering communication and cooperation among logicians of diverse backgrounds.

Format The ajL publishes articles which further research in the field. We actively encourage submissions of papers reporting original research, as well as expository articles intended to summarise a field for the interested reader, and reviews giving critical commentary on recent books and articles. Each submission to the journal undergoes a rigorous refereeing procedure, ensuring 
that all articles published meet high standards of originality, significance and clarity.

\section{WHY IS IT?}

Why ANOTHER LOGIC JOURnAL? There are many very good logic journals published throughout the world. However, no general logic journal is produced in Australia or New Zealand, despite the long and active tradition we have in research in logic. We (the Australasian Association for Logic) have often wished to produce a journal covering our many areas of interest, and now the technology is at a stage at which this is not only possible but feasible.

Why an electronic Journal? The last decade of the 2oth Century and the beginning of the 2Ist has a combination of a sharp rise in the cost of print journals, and a sharp decline in the purchasing power of many university libraries. As a result, research collections in many universities are shrinking and not expanding. This places great pressure on the fabric of our research culture: many people who would like to keep up with current research simply cannot afford to do so.

At the same time as costs rise and budgets shrink, the technologies of electronic document production and delivery are developing quickly, so we have alternatives. Electronic journals provide a means for efficient, affordable and widespread delivery of research. Not only is the $\mathrm{AJL}$ available free to subscribers who know how to look for issues of the journal (or who have abstracts delivered in their email), but since the journal is freely available on the web, search engines index all of our papers and provide many more ways for readers to come and find current research.

\section{Who ARE WE?}

Who runs the journal? The ajl is published by the Australasian Association for Logic: a loose collection of researchers in logic throughout Australia and New Zealand. Our chief activity is our annual conference. The journal is managed by a small editorial board, and its work is overseen by the Association as a whole. Many researchers, throughout Australasia and abroad help the journal by working as referees, providing feedback on papers submitted for publication.

Who can submit to the journal? Anyone can submit articles for publication to the journal. Just follow the instructions for authors $\breve{0}$ for details of how to prepare your manuscript for submission. If your submission falls within the scope of the journal, we will send your article off for prompt refereeing. If the reports are favourable, and if the editorial board agrees that the paper is fit for publication, we will then work with you in the copyediting phase to produce the final manuscript, which is published here as soon as it is ready. 


\section{When is it PUBLISHED?}

What is the publication schedule? One advantage of an electronic journal is the publication timetable. All communication between author, editors and referees occurs by email, and so the submit/referee/correct/acceptor-reject cycle is shortened. (Admittedly, this can happen with print journals too, but not many print journals in logic accept electronic submission of manuscripts.) The other advantage is at the point the manuscript is ready for publication. Each individual article may be published online whenever it is ready. It need not wait for the rest of the volume to appear. "Volumes" are finalised at the end of each calendar year, but their contents appear when they are ready.

\section{How DOES IT WORK?}

ACKNOwLEDGEMENTS This enterprise is only possible because of the efforts of many people. We thank the University of Melbourne Philosophy Department for server space, access to computers and infrastructure, and administrative support. We thank the logic community in Australasia and worldwide for refereeing support. And of course we thank the authors for writing! 
The Australasian fournal of Logic (ISSN: 1448-5052) disseminates articles that significantly advance the study of logic, in its mathematical, philosohpical or computational guises. The scope of the journal includes all areas of logic, both pure and applied to topics in philosophy, mathematics, computation, linguistics and the other sciences.

Articles appearing in the journal have been carefully and critically refereed under the responsibility of members of the Editorial Board. Only papers judged to be both significant and excellent are accepted for publication.

The journal is freely available at the journal website at

$$
\text { http://www.philosophy.unimelb.edu.au/ajl/ }
$$

All issues of the journal are archived electronically at the journal website.

SUBSCRIPTIONS Individuals may subscribe to the journal by sending an email, including a full name, and email address and a postal address to the managing editor at ajl-editors@unimelb.edu.au Subscribers will receive email abstracts of accepted papers to an address of their choice. For institutional subscription, please email the managing editor at ajl-editors@unimelb.edu.au

Complete published papers may be downloaded at the journal's website at http: //www.philosophy.unimelb.edu.au/ajl/ The journal currently publishes in pdf format.

Submission The journal accepts submissions of papers electronically. To submit an article for publication, send the $\mathrm{LT}_{\mathrm{E}} \mathrm{X}$ source of a submission to a member of the editorial board. For a current list of the editorial board, consult the website.

The copyright of each article remains with the author or authors of that article. 